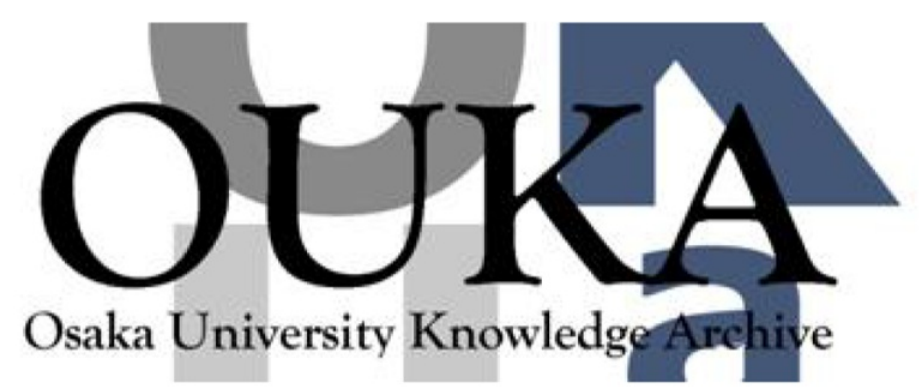

\begin{tabular}{|c|l|}
\hline Title & $\begin{array}{l}\text { Hairpin vortices in the largest scale of } \\
\text { turbulent boundary layers }\end{array}$ \\
\hline Author(s) & Motoori, Yutaro; Goto, Susumu \\
\hline Citation & $\begin{array}{l}\text { International Journal of Heat and Fluid Flow. } \\
86 \text { p. 108658 }\end{array}$ \\
\hline Issue Date & $2020-12$ \\
\hline oaire:version & AM \\
\hline URL & https://hdl. handle. net/11094/77681 \\
\hline rights & \\
\hline Note & \\
\hline
\end{tabular}

Osaka University Knowledge Archive : OUKA

https://ir. Library. osaka-u. ac. jp/

Osaka University 


\title{
Hairpin vortices in the largest scale of turbulent boundary layers
}

\author{
Yutaro Motoori*, Susumu Goto** \\ Graduate School of Engineering Science, Osaka University, 1-3 Machikaneyama, Toyonaka, Osaka, 560-8531, Japan
}

\section{ARTICLE INFO}

\section{Keywords:}

Direct numerical simulation

Turbulent boundary layer

Hairpin vortex

\begin{abstract}
A B S T R A C T
We have conducted direct numerical simulations of a turbulent boundary layer for the momentum-thickness-based Reynolds number $R e_{\theta}=180-4600$. To extract the largestscale vortices, we coarse-grain the fluctuating velocity fields by using a Gaussian filter with the filter width comparable to the boundary layer thickness. Most of the largestscale vortices identified by isosurfaces of the second invariant of the coarse-grained velocity gradient tensor are similar to coherent vortices observed in low-Reynolds-number regions, that is, hairpin vortices or quasi-streamwise vortices inclined to the wall. We also develop a percolation analysis to investigate the threshold-dependence of the isosurfaces and objectively identify the largest-scale hairpin vortices in terms of the coarsegrained vorticity, which leads to the quantitative evidence that they never disappear even in fully developed turbulent regions. Hence, we conclude that hairpin vortices exist in the largest-scale structures irrespective of the Reynolds number.
\end{abstract}

\section{Introduction}

It is now a common sense that there exist coherent structures even in developed turbulence. Since coherent structures are a key to understanding the dynamics of turbulence, it is desired to reveal what kind of structure is dominant. For example, quasi-streamwise vortices, which are inclined to the wall-normal and spanwise directions, are dominant structures in the buffer layer of wall-bounded turbulence. Direct numerical simulations (DNS) of turbulent channel flow (e.g. Jiménez and Moin, 1991; Jeong et al., 1997) and turbulent boundary layers (e.g. Spalart, 1988; Robinson, 1991) significantly contributed to understanding the turbulence in terms of the coherent structures. In addition to the quasi-streamwise vortices, it is also known that hairpinshaped vortices exist in turbulent boundary layers. Figure 1 shows typical hairpin vortices which are induced by an artificial body force in the present DNS (see Section 2.1 for the details). Their downstream part is lifted up from the wall and orients to the spanwise direction to form hairpin vortices. We emphasize that these hairpin vortices are not numerical artifacts, but in fact, many experiments (e.g. Kline et al., 1967; Falco, 1977; Bandyopadhyay, 1980; Head and Bandyopadhyay, 1981; Smith et al., 1991) also showed the existence of hairpin vortices in turbulent boundary layers, and many models and theories have been developed to explain the statistics of the turbulence in terms of hairpin vortices (e.g. Theodorsen, 1952, 1955; Townsend, 1976; Perry and Chong, 1982; Perry et al., 1986; Perry and Marusic, 1995; Hwang, 2015; Marusic and Monty, 2019).

It is then natural to examine whether these coher-

\footnotetext{
${ }^{*}$ Corresponding author. Tel.: +81668506190 .

y_motoori@fm.me.es.osaka-u.ac.jp (Y. Motoori); goto@me.es.osaka-u.ac.jp (S. Goto)

ORCID(s): 0000-0002-9647-9014 (Y. Motoori); 0000-0001-7013-7967 (S. Goto)
}

ent vortices (i.e. quasi-streamwise vortices and hairpin vortices) are also dominant in developed turbulence. To investigate this issue, Adrian et al. (2000) examined the two-dimensional velocity fields on the spanwise plane which are obtained by particle image velocimetry (PIV). Their study found hairpin vortex signatures (HVS) which are described by the combination of flow elements: a swirling region, an ejection, a stagnation point between the ejection and an upstream sweep, and a low-speed region near the wall (see Fig. 10 in Adrian et al., 2000). The swirling region corresponds to the head of a hairpin vortex, whereas the ejection corresponds to the upflow in the low-speed region between the two legs and the ejection collides with the sweep from the upstream to form a stagnation point. According to the PIV studies (e.g. Adrian et al., 2000; Ganapathisubramani et al., 2003; Adrian, 2007; Dennis and Nickels, 2011a) on the turbulent boundary layers at high Reynolds numbers, the HVS are frequently to form a so-called hairpin packet in the streamwise direction.

For the last decade, quite a few authors investigated the details of three-dimensional vortical structures by using DNS of turbulent boundary layers. For example, the visualization of small-scale vortices by $\mathrm{Wu}$ and Moin $(2009 a, b)$ showed the forest of hairpins in an upstream region with low Reynolds numbers, where the effect of the transition remains. The result is consistent with those by the experiments (e.g. Kline et al., 1967; Falco, 1977; Bandyopadhyay, 1980; Head and Bandyopadhyay, 1981; Smith et al., 1991; Adrian et al., 2000; Ganapathisubramani et al., 2003; Adrian, 2007; Dennis and Nickels, 2011a). On the other hand, it was also reported that, as the Reynolds number increases, vortical structures viewed from the top (i.e. vortices in the $\log$ or outer layers) are not hairpin-shaped (Jiménez et al., 2010; Schlatter et al., 2014; Eitel-Amor et al., 2015). Although arch-like vortices are observed around 
the boundary layer thickness (i.e. the turbulent/nonturbulent interface) in these DNS, Schlatter et al. (2014) and Rahgozar and Maciel (2016) showed that most of the spanwise vorticity, which is an element of the HVS, does not correspond to the head of a hairpin but arch-like vortex without legs attached to the wall. In summary, these studies of the DNS of high-Reynoldsnumber turbulent boundary layers concluded that the spanwise vorticity is not a sufficient condition for the existence of hairpin vortices and that the smallest-scale vortices in the $\log$ and outer layers are not hairpinshaped. This seems to be conflicting with the experimental results where the hairpin vortices are observed as the HVS. A purpose of the present study is to get rid of this superficial inconsistency. Although it is not conclusive whether or not hairpin vortices exist in the buffer layer of the developed turbulent boundary layers (e.g. Schlatter et al., 2014; Jodai and Elsinga, 2016), the targets of the present study are vortices larger than them, that is, boundary-layer-thickness-scale vortices.

Noting that the magnitude of the velocity gradient in turbulence is determined mainly by the smallest-scale structures, vortices identified by the DNS (e.g. Jiménez et al., 2010; Schlatter et al., 2014) are at the smallest scale. On the other hand, the velocity is determined by the largest-scale structures. Since the HVS is based on the structures (ejections, sweeps and low-speed regions) related to the velocity, the observed structures are related to the largest-scale vortices. Therefore, we can infer that vortices observed in the DNS and experiments are at different scales. This inference is consistent with our previous DNS study (Motoori and Goto, 2019a), where we identified the hierarchy of multiscale vortices by coarse-graining the velocity fields at different scales. Our visualization of the coarse-grained turbulent fields qualitatively showed that the largest-scale vortices are likely to be hairpin vortices (which correspond to the HVS in the experiments), whereas the smaller-scale vortices in the log layer are randomly oriented [which correspond to vortices in the DNS by Jiménez et al. (2010) and Schlatter et al. (2014)]. The observation of the largestscale hairpin vortices is also consistent with the studies (e.g. Ganapathisubramani et al., 2003; Lee and Sung, 2011; Lee et al., 2014) on (very) large-scale motions (LSM). For example, Lee and Sung (2011) identified LSM with the filtered velocity to show that they are related to the large-scale hairpin vortices. Thus, filtering is necessary to identify large-scale structures, which was used by many authors (e.g. Abe et al., 2004; Hutchins and Marusic, 2007; Elsinga et al., 2010; Lee and Sung, 2011; Lee et al., 2014, 2015, 2017; Hwang et al., 2016; Lozano-Durán et al., 2016; Deshpande et al., 2019; Kevin et al., 2019a,b). Incidentally, del Álamo et al. (2006) and Dennis and Nickels (2011a) extracted largescale vortical structures by conditionally averaging the turbulent fields, which may be regarded as a kind of

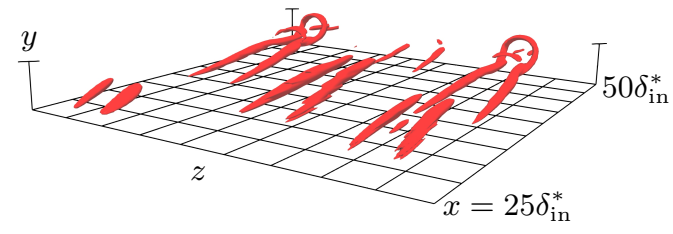

Fig. 1: Hairpin vortices induced by the body force. Isosurfaces of the second invariant of the velocity gradient tensor (with the threshold $Q^{+}=0.1$ ) are shown. The grid width indicates the boundary thickness at the exit plane of this visualization.

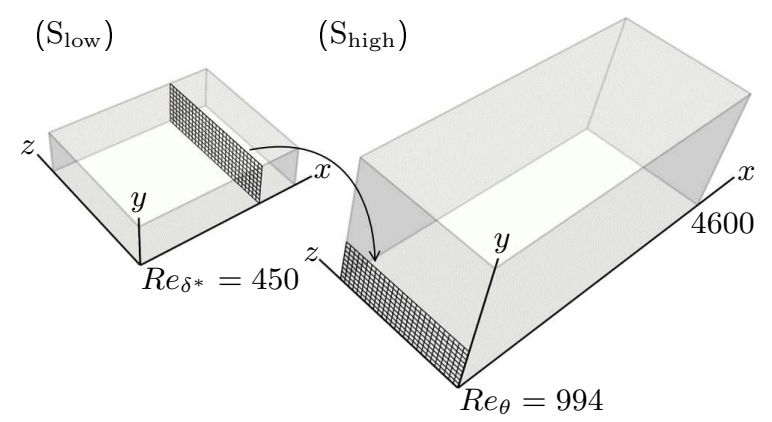

Fig. 2: Schematic of the computational domains of the present two DNS. The inlet condition of the simulation $\left(\mathrm{S}_{\text {high }}\right)$ for higher Reynolds numbers is the time-series of turbulent fields at the streamwise location $\left(R e_{\theta}=994 ; x=3 L_{x} / 4\right)$ obtained by the simulation ( $\mathrm{S}_{\text {low }}$ ) for lower Reynolds numbers.

coarse-graining.

In the present study, we conduct DNS of a streamwisely-elongated turbulent boundary layer for the momentum-thickness-based Reynolds number $R e_{\theta}=180-4600$. We focus on vortices at the largest scale comparable to the boundary layer thickness so that we can quantitatively show the dominance of hairpin vortices and its Reynolds-number independence. For this purpose, we apply a Gaussian filter with the filter width comparable to the boundary layer thickness. In the rest of the present paper, we first describe the methods of the DNS (Section 2.1) and coarse-graining (Section 2.2). We then objectively identify the largestscale vortices (Section 3.1) to quantitatively show that hairpin vortices exist in the largest-scale structures irrespective of the Reynolds number (Section 3.2). Then, we will show that the present result is not conflicting with the previous numerical and experimental results (Section 4).

\section{Methods}

\subsection{Direct numerical simulation}

We numerically simulate turbulent boundary layers with zero pressure gradient over a flat plate by solving the Navier-Stokes equations of an incompressible fluid by a standard finite difference scheme. Although the 
Table 1

Numerical parameters of the DNS for the low ( $\mathrm{S}_{\text {low }}$ ) and high ( $\mathrm{S}$ high $)$ Reynolds numbers. Here, $L_{x}, L_{y}$ and $L_{z}$ are the sides of the computational domain, $N_{x}, N_{y}$ and $N_{z}$ are the numbers of the grid points, and $\Delta x^{+}, \Delta y_{\min }^{+}$ (the smallest grid width) and $\Delta z^{+}$are the resolutions at the domain exit. $\delta_{\text {in }}^{*}$ is the displacement thickness at the inlet of $\mathrm{S}_{\text {low }}$.

\begin{tabular}{c|cccc}
\hline & $\left(L_{x}, L_{y}, L_{z}\right)$ & $\left(N_{x}, N_{y}, N_{z}\right)$ & $\left(\Delta x^{+}, \Delta y_{\min }^{+}, \Delta z^{+}\right)$ & $R e_{\theta}$ \\
\hline$S_{\text {low }}$ & $(1000,80,240) \delta_{\text {in }}^{*}$ & $(2048,384,1024)$ & $(9.7,0.18,4.6)$ & $180-1190$ \\
$S_{\text {high }}$ & $(5000,240,240) \delta_{\text {in }}^{*}$ & $(8064,640,768)$ & $(10,0.29,5.2)$ & $990-4600$ \\
\hline
\end{tabular}

(a)

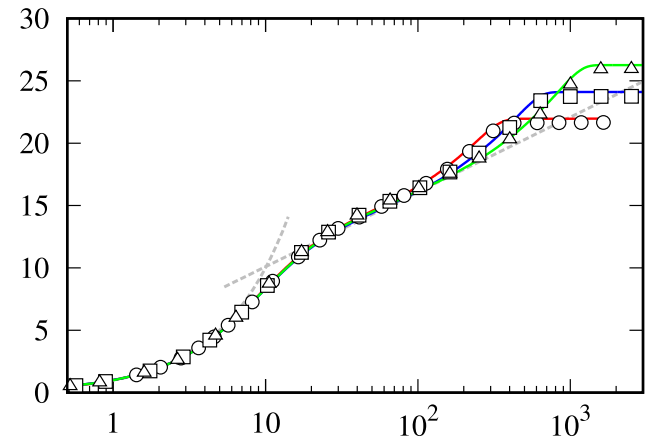

(b)

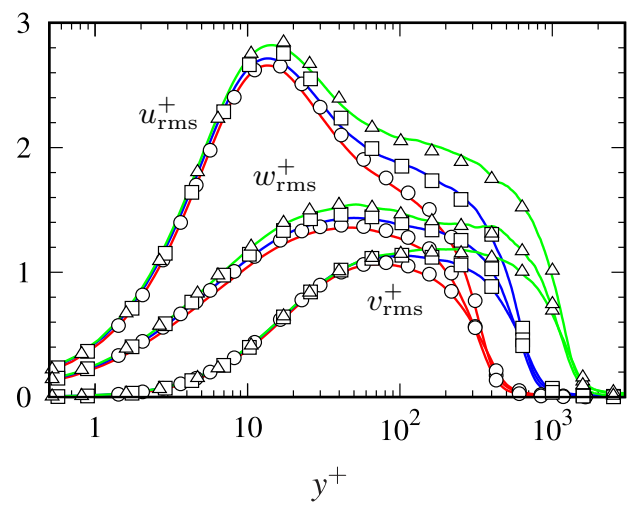

Fig. 3: Wall-normal distributions of (a) the mean streamwise velocity and (b) the root-mean-square values of the fluctuating velocity components. Lines show the present results at the locations with $R e_{\theta}=1000$ (red), 2000 (blue) and 4060 (green), respectively. The open circles, squares and triangles indicate the results by Schlatter and Örlü (2010) for the same Reynolds numbers $R e_{\theta}=1000,2000$ and 4060. In (a), the grey dashed lines indicate the law of the wall: $U^{+}=y^{+}$and $U^{+}=(1 / \kappa) \ln \left(y^{+}\right)+B$ with $\kappa=0.384$ and $B=4.1$.

main program for the present DNS is identical to the one in our previous study (Motoori and Goto, 2019a), we change the boundary conditions at the inlet. For the previous inlet condition, we used the time-series data of fully developed turbulent velocity fields provided by Lee et al. (2013, 2017). Here, as shown in Fig. 2, we separately simulate the low- and high-Reynoldsnumber turbulence for reducing the required computational resources. The inlet condition for the former low-Reynolds-number simulation $\left(\mathrm{S}_{\text {low }}\right)$ is the Blasius solution and the body force is imposed at the streamwise location $x=25 \delta_{\text {in }}^{*}$, where $\delta_{\text {in }}^{*}$ is the displacement

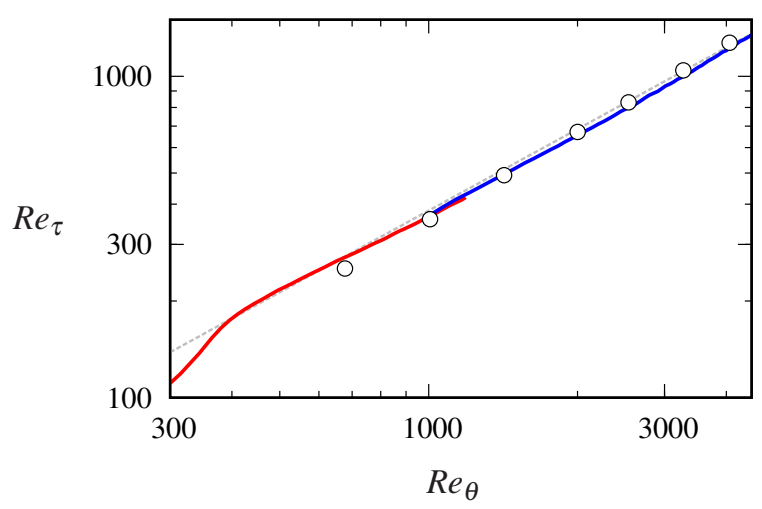

Fig. 4: Relation between the Reynolds numbers $R e_{\theta}$ and $R e_{\tau}$. The red line is the results of the low-Reynolds-number simulation $\left(\mathrm{S}_{\text {low }}\right)$ and blue one is those of the high-Reynoldsnumber simulation $\left(\mathrm{S}_{\text {high }}\right)$. The open circles are the results by Schlatter and Örlü (2010). The gray dashed line indicates $R e_{\tau}=1.13 \times R e_{\theta}^{0.843}$, which is the best fit to the databases of Schlatter and Örlü (2010).

thickness at the inlet plane. As the forcing, we employ the method used by Eitel-Amor et al. (2015), but we use a smaller value $0.096 U_{\infty}^{2} / \delta_{\text {in }}^{*}$ for the parameter $C_{2}$ of the amplitude of the force in the wall-normal direction than the value used by Eitel-Amor et al. (2015). However, we observe in Fig. 1 hairpin vortices which are identified by the isosurfaces of the second invariant $Q$ of the velocity gradient tensor. On the other hand, as the inlet condition for the high-Reynolds-number simulation $\left(\mathrm{S}_{\text {high }}\right)$, we use the time series-data of the velocity fields obtained by $\mathrm{S}_{\text {low }}$ at the streamwise location $x=3 L_{x} / 4$ corresponding to $R e_{\theta}=994$. Here, $L_{x}$ is the streamwise length of the computational domain of $\mathrm{S}_{\text {low }}$. The other boundary conditions and numerical schemes of these two simulations are the same as in our previous study (Motoori and Goto, 2019a). The resolution is also set to be similar (Table 1) by using a sufficiently large number of grid points. Here, $x, y$ and $z$ denote the streamwise, wall-normal and spanwise directions, respectively, and .+ denotes the wall units defined in terms of the skin-friction velocity $u_{\tau}$ and the kinematic viscosity $\nu$.

We show in Fig. 3 the wall-normal distributions of (a) the mean streamwise velocity $U$ and (b) standard deviation $\left(u_{\mathrm{rms}}, v_{\mathrm{rms}}\right.$ and $\left.w_{\mathrm{rms}}\right)$ of the streamwise, 
(a)

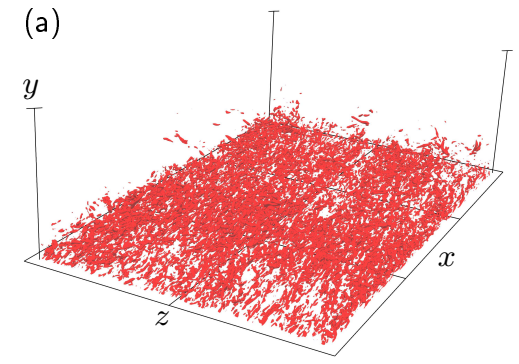

(b)
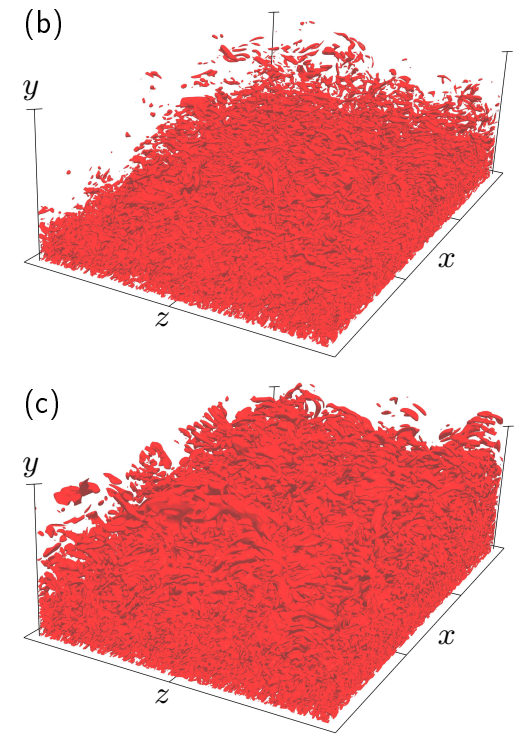

Fig. 5: Small-scale vortices identified by the isosurfaces of the second invariant $Q$ of the velocity gradient tensor in the region $3680 \leq \operatorname{Re}_{\theta} \leq 3780$. The thresholds are (a) $Q^{+}=1.0 \times 10^{-2}$, (b) $1.0 \times 10^{-3}$ and (c) $1.0 \times 10^{-4}$. The grid width indicates the boundary thickness at the exit plane of the visualization.

wall-normal and spanwise velocity for $R e_{\theta}=1000$ (red lines), 2000 (blue lines) and 4060 (green lines). Our results are in good agreement with those (open symbols) of the DNS by Schlatter and Örlü (2010). We also show in Fig. 4 the relation between $R_{\theta}$ and the friction Reynolds number $R e_{\tau}$ (red line, $S_{\text {low }}$; blue line, $\mathrm{S}_{\text {high }}$ ), which is also in agreement with the results by Schlatter and Örlü (2010).

\subsection{Coarse-graining}

To extract the largest-scale vortices in the turbulent boundary layers, we coarse-grain the simulated turbulent fields. More concretely, we apply a threedimensional Gaussian filter

$$
\begin{aligned}
u_{i}^{(\sigma)}(\boldsymbol{x})=C \int_{V} & \check{u}_{i}\left(\boldsymbol{x}^{\prime}\right) \\
& \quad \exp \left(-\frac{2}{\sigma^{2}}\left(\boldsymbol{x}-\boldsymbol{x}^{\prime}\right)^{2}\right) \mathrm{d} \boldsymbol{x}^{\prime}
\end{aligned}
$$

to $\check{u}_{i}\left(\boldsymbol{x}^{\prime}\right)\left(=u_{i}\left(\boldsymbol{x}^{\prime}\right)-\left\langle u_{i}\left(\boldsymbol{x}^{\prime}\right)\right\rangle_{z}\right)$, that is the fluctuating velocity $u_{i}\left(\boldsymbol{x}^{\prime}\right)$ minus the spanwisely-averaged velocity $\left\langle u_{i}\left(\boldsymbol{x}^{\prime}\right)\right\rangle_{z}$. Here, $C$ is a constant to ensure that the integration of the filter gives unity, $\sigma$ is the filter width
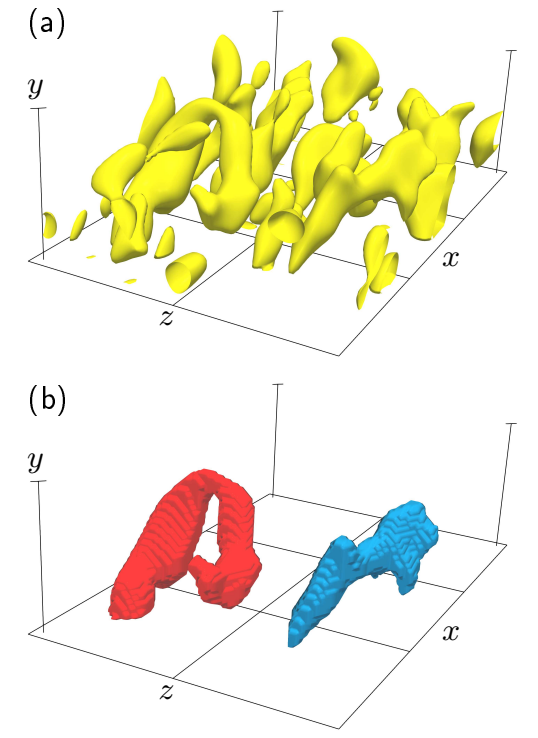

Fig. 6: (a) Largest-scale vortices identified by the isosurfaces of the second invariant $Q^{(\sigma)}$ of the velocity gradient tensor coarse-grained at $\sigma^{*}=0.2$. The threshold is $Q^{(\sigma) *}=16$. The instance and location are the same as in Fig. 5. (b) A hairpin vortex (red) and a quasi-streamwise vortex inclined to the wall (blue) are extracted from the field shown in (a).

and $\langle\cdot\rangle_{z}$ is the spanwisely-averaged quantity. For the wall-normal direction, we employ the method proposed by Lozano-Durán et al. (2016) that the filtering operation is extended by reflecting the filter at the wall and the sign of the wall-normal velocity $\check{u}_{2}(=\check{v})$ is inverted in order to ensure the incompressibility and the no-slip boundary condition of $v^{(\sigma)}$. This filter may correspond to the low-pass filter of the Fourier modes of the velocity, and the velocity field $u_{i}^{(\sigma)}$ coarse-grained at $\sigma$ has only the information larger than $\sigma$.

To focus on the boundary-layer-thickness-scale vortices, we fix the filter width $\sigma^{*}=\sigma / \delta_{99}=0.2$, where $\delta_{99}$ denotes the boundary layer thickness and ${ }^{*}$ is the outer scale defined in terms of $u_{\tau}$ and $\delta_{99}$. Note that the filter width is a function of the streamwise location $x$. Note also that, near the wall in high-Reynolds-number regions, hairpin vortices with scales $\left(y \ll \sigma \approx \delta_{99}\right)$ much smaller than the boundary layer thickness, which were observed by Jodai and Elsinga (2016), are filtered out. In our previous studies (Motoori and Goto, 2019a,b), we referred to structures with $\sigma \sim y$ as the largestscale structures, whereas in the present study we refer to structures with $\sigma \sim \delta_{99}$ as the largest-scale ones.

\section{Results}

\subsection{Identification of the largest-scale vortices}

When we visualize the isosurface of a quantity related to the velocity gradient without coarse-graining, we always observe only structures at the smallest scale. 
Hairpin vortices in the largest scale of TBL

Table 2

Range of the Reynolds numbers in the six streamwise regions (R1-R6).

\begin{tabular}{c|cccccc}
\hline & $\mathrm{R} 1\left(\mathrm{~S}_{\text {low }}\right)$ & $\mathrm{R} 2\left(\mathrm{~S}_{\text {low }}\right)$ & $\mathrm{R} 3\left(\mathrm{~S}_{\text {high }}\right)$ & $\mathrm{R} 4\left(\mathrm{~S}_{\text {high }}\right)$ & $\mathrm{R} 5\left(\mathrm{~S}_{\text {high }}\right)$ & $\mathrm{R} 6\left(\mathrm{~S}_{\text {high }}\right)$ \\
\hline$R e_{\theta}$ & $480-750$ & $750-990$ & $990-1950$ & $1950-2900$ & $2900-3780$ & $3780-4600$ \\
$x / L_{x}$ & $\frac{1}{4}-\frac{1}{2}$ & $\frac{1}{2}-\frac{3}{4}$ & $0-\frac{1}{4}$ & $\frac{1}{4}-\frac{1}{2}$ & $\frac{1}{2}-\frac{3}{4}$ & $\frac{3}{4}-1$ \\
\hline
\end{tabular}

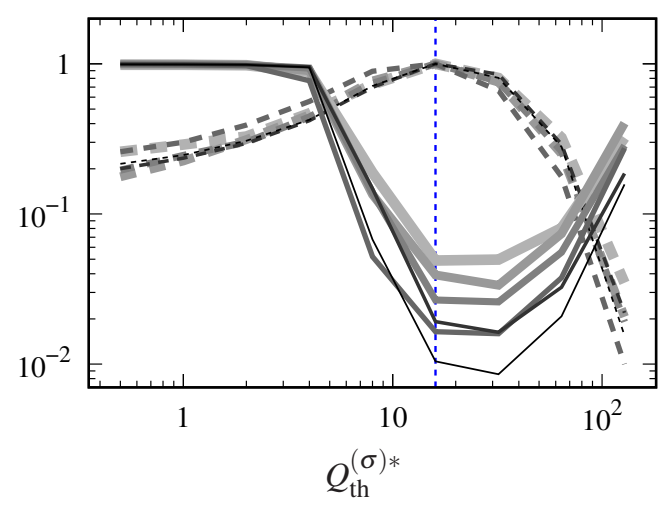

Fig. 7: Percolation analyses for the largest-scale vortices $\left(Q^{(\sigma)} \geq Q_{\text {th }}^{(\sigma)}\right.$ at $\left.\sigma^{*}=0.2\right)$. The dashed lines show the ratio $N / N_{\text {max }}$ of the number of identified objects to their maximum number. The solid lines show the ratio $V_{\text {lar }} / V_{\text {tot }}$ of the volume of the largest object to the total volume of all identified objects. The thickness and darkness indicate the streamwise location (The thinnest/darkest line is for R1; the thickest/lightest is for R6; see Table 2). The blue vertical line indicates $Q_{\mathrm{th}}^{(\sigma) *}=16$.

This is because the magnitude of the velocity gradient is mainly determined by the smallest-scale structures. For example, Fig. 5 shows the isosurfaces of the second invariant $Q$ of the velocity gradient tensor in the region $3680 \leq \operatorname{Re}_{\theta} \leq 3780$. As we decrease the threshold [(a) $Q^{+}=1.0 \times 10^{-2}$, (b) $1.0 \times 10^{-3}$ and (c) $1.0 \times 10^{-4}$ ], we observe more vortices away from the wall, which tend to connect to each other. We emphasize that the radius of the identified tubular vortices is much smaller than the grid width in the figure (i.e. the boundary layer thickness at the visualized exit plane). In addition, vortices near the boundary layer thickness tend to align to the spanwise direction (Fig. 5c), that is, they form arch-like vortices. This tendency is consistent with the visualizations of vortices by Jiménez et al. (2010) and Schlatter et al. (2014), for which they used the $\Delta$-method (which was proposed by Chong et al., 1990) and the $\lambda_{2}$-method (which was proposed by Jeong and Hussain, 1995), respectively.

In order to extract the largest-scale vortices, we use the second invariant $Q^{(\sigma)}$ of the velocity gradient tensor evaluated from the velocity field $u_{i}^{(\sigma)}$ coarse-grained at $\sigma^{*}=0.2$. Figure 6(a) shows the isosurfaces of $Q^{(\sigma)}$ and we see that the sizes of the identified vortices are comparable to the boundary layer thickness indicated by
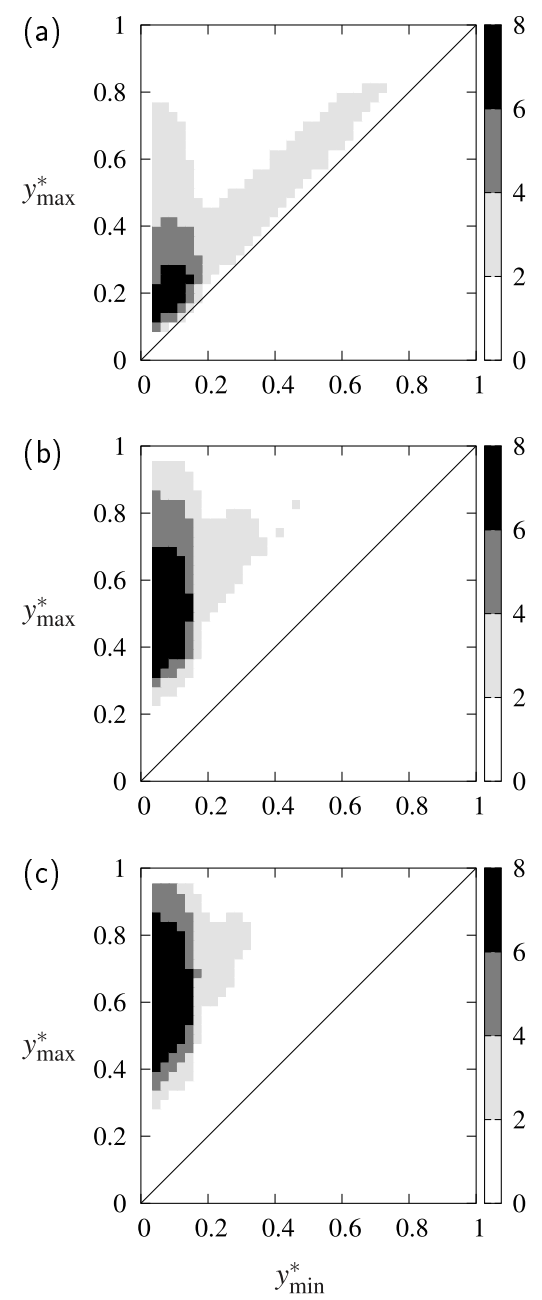

Fig. 8: Joint probability density functions of the minimum $y_{\min }^{*}$ and maximum $y_{\max }^{*}$ distance from the wall of (a) all objects, (b) the larger ones satisfying $V^{*} \geq 0.01$ and (c) $V^{*} \geq 0.02$ identified with $Q^{(\sigma) *}=16$ for all regions (R3-R6) of Shigh.

the grid width in the figure. More precisely, the radius of the tubular vortices is approximately the filter scale $\sigma^{*}=0.2$ and the longitudinal length is comparable to the boundary layer thickness. Moreover, looking at the shapes of the largest-scale vortices, we notice that they are similar to the dominant coherent structures in low-Reynolds-number turbulence, namely, a hairpin vortex or a quasi-streamwise vortex (see in Fig. 6b two vortices extracted from Fig. 6a). Note that these vortices are attached to the wall, but their legs are differ- 
ent from buffer-layer coherent vortices, which are absent in the filtered field. Thus, the visualization leads us to infer that hairpin vortices (and quasi-streamwise vortices) do not exist as small-scales structures in the $\log$ and outer layers of high-Reynolds-number turbulence [Fig. 5, Jiménez et al. (2010) and Schlatter et al. (2014)], but they do exist as the largest-scale structures. We can also see that the largest-scale vortices do not correspond to the clusters of the small-scale vortices (Fig. 5). This is because small-scale vortices are created in the larger-scale straining fields around the large-scale vortices in the log layer (Motoori and Goto, 2019a).

As observed in Fig. 5, vortical regions identified by isosurfaces depend on the threshold. Here, to objectively determine the threshold $Q_{\mathrm{th}}^{(\sigma)}$ for the identification of the largest-scale vortices, we investigate the percolation of the region satisfying the condition $Q^{(\sigma)} \geq$ $Q_{\mathrm{th}}^{(\sigma)}$ by using the computational algorithm proposed by Hoshen and Kopelman (1976). More concretely, we tag the same label to the neighboring connected grid cells satisfying $Q^{(\sigma)} \geq Q_{\text {th }}^{(\sigma)}$ to count the number of individual vortices for different values of $Q_{\mathrm{th}}^{(\sigma)}$. The dashed curves in Fig. 7 show the number normalized by its maximum. The thickness and darkness of the lines indicate the streamwise location, and we see that their trends are independent of the location (i.e. the Reynolds number). For example, when the threshold is small $\left(Q_{\mathrm{th}}^{(\sigma) *} \lesssim 4\right)$, the number is much smaller than its maximum because many of the objects are connected. As increasing the threshold $\left(10 \lesssim Q_{\mathrm{th}}^{(\sigma) *} \lesssim 30\right)$, since the identified objects are separated, the number of individual vortices becomes the largest at about $Q_{\mathrm{th}}^{(\sigma) *}=16$ (blue vertical line). For $Q_{\mathrm{th}}^{(\sigma) *} \gtrsim 100$, since the objects shrink, the number of them again decreases. This trend of the number of the objects is consistent with the ratio $V_{\text {lar }} / V_{\text {tot }}$ (solid lines) of the volume of the largest object $V_{\text {lar }}$ to the total volume $V_{\text {tot }}$ of all objects. Namely, the volume ratio is the smallest around $Q_{\mathrm{th}}^{(\sigma) *}=16$ because the number of the objects is the largest. This is the reason why, in the present study, we use the isosurfaces with the threshold $Q_{\mathrm{th}}^{(\sigma) *}=16$.

Before quantitatively showing the dominance of hairpin vortices, we investigate the size of the objects identified by the objective threshold $\left(Q_{\mathrm{th}}^{(\sigma) *}=16\right)$. Figure $8(\mathrm{a})$ shows the joint probability density function (PDF) of the minimum height $y_{\min }$ and the maximum height $y_{\max }$ within each object evaluated in $\mathrm{S}_{\text {high }}$ (i.e. R3-R6; see Table 2). We can see two regions with higher values of the PDF. One is the vertical band in $0 \lesssim y_{\text {min }}^{*} \lesssim 0.2$ which stems from vortices attached to the wall, and the other is the diagonal band with $y_{\max }^{*} \approx y_{\min }^{*} \gtrsim 0.2$ which corresponds to small-scale objects detached to the wall. Since we examine the coarse-grained turbulent fields, the detached objects are not small-scale vortices but larger than $\sigma\left(=0.2 \delta_{99}\right)$.
It is interesting that the similar behavior of the joint PDF was observed for other quantities without coarsegraining (del Álamo et al., 2006; Lozano-Durán et al., 2012; Osawa and Jiménez, 2018).

We show in Fig. 8(b) the joint PDF for the larger objects satisfying the condition for the volume $V^{*} \geq$ 0.01 . We see that this condition gets rid of the group formed by detached objects $\left(y_{\min }^{*} \approx y_{\max }^{*}\right)$ and highlights only the group formed by the attached vortices $\left(0 \lesssim y_{\min }^{*} \lesssim 0.2\right.$ and $\left.0.2 \lesssim y_{\max }^{*} \lesssim 1\right)$. As increasing the value of the condition to $V^{*} \geq 0.02$, attached vortices are more highlighted (Fig. 8c).

In the next section, by using the objective threshold $\left(Q^{(\sigma) *}=16\right)$ based on the percolation analyses and these conditions ( $V^{*} \geq 0.01$ or 0.02 ), we will quantitatively show the dominance of hairpin vortices and quasi-streamwise vortices.

\subsection{Largest-scale hairpin and quasi-streamwise vortices}

We show in Fig. 9 the isosurfaces with the threshold $Q^{(\sigma) *}=16$ in the streamwisely-elongated regions (a) R3 $\left(990 \leq R e_{\theta} \leq 1950\right)$ and (b) R5 $\left(2900 \leq R e_{\theta} \leq\right.$ 3780 ). Irrespective of the streamwise location, we can find hairpin vortices, one-legged vortices and quasistreamwise vortices inclined to the wall. These inclination angles to the wall of the legs of hairpin vortices and quasi-streamwise vortices are approximately 45 degrees, which is consistent with the results by Deshpande et al. (2019) and reference therein. This is because they are stretched mainly by the mean shear (Motoori and Goto, 2019a), whose stretching direction in the log layer is identical to the orientation of the legs.

By quantitatively identifying hairpin vortices and quasi-streamwise vortices in the direction of the meanflow stretching, we here investigate their dominance irrespective of the Reynolds number. For this purpose, we identify them in terms of the coarse-grained vorticity. Hairpin vortices are composed of two legs with opposite signs of the streamwise vorticity and of a head with the spanwise vorticity near the boundary layer thickness. To define a hairpin vortex, we look for, in each connected region satisfying $Q^{(\sigma) *} \geq 16$, the maxi$\operatorname{mum} \omega_{x}^{(\max )}$ and minimum values $\omega_{x}^{(\min )}$ of the streamwise vorticity and the minimum value $\omega_{z}^{\left(\min \mid y^{*}>0.5\right)}$ of spanwise vorticity for $y^{*}>0.5$. Here, we omit the superscript.$^{(\sigma)}$ for the vorticity, though it is evaluated from the coarse-grained velocity $u_{i}^{(\sigma)}$. We then call the connected region a hairpin vortex when it satisfies the following conditions:

$$
\begin{aligned}
& \omega_{x}^{(\max )}>0, \omega_{x}^{(\min )}<0, \omega_{z}^{\left(\min \mid y^{*}>0.5\right)}<0, \\
& z\left(\omega_{x}^{(\max )}\right)-z\left(\omega_{x}^{(\min )}\right)>2 \sigma\left(=0.4 \delta_{99}\right), \\
& z\left(\omega_{x}^{(\max )}\right)>z\left(\omega_{z}^{\left(\min \mid y^{*}>0.5\right)}\right)>z\left(\omega_{x}^{(\min )}\right),
\end{aligned}
$$

where $z(\cdot)$ denotes the spanwise location of a quantity $(\cdot)$. The first condition (2a) implies a necessary 


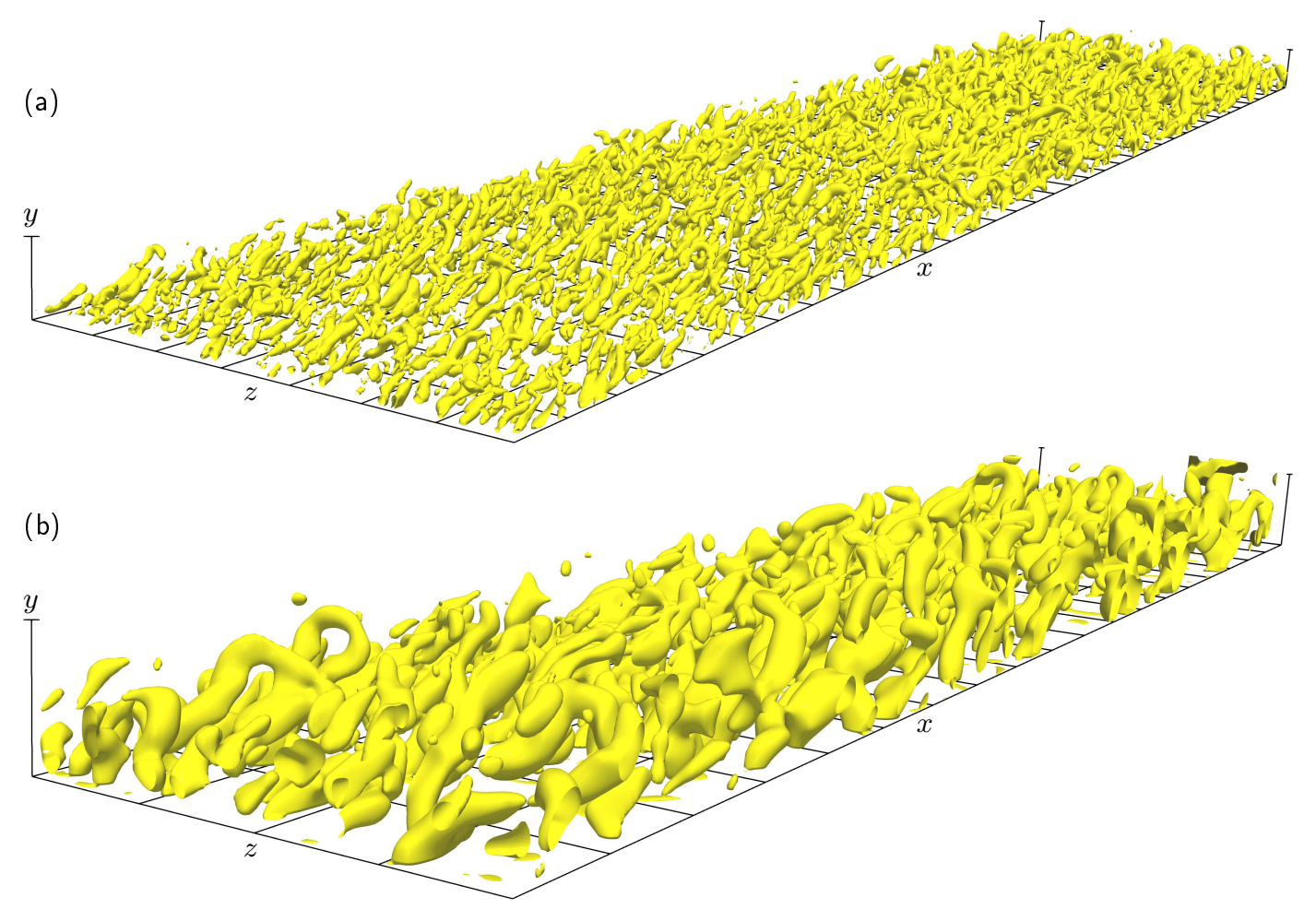

Fig. 9: Visualizations in long regions [(a) R3 and (b) R5 in Table 2] of the largest-scale vortices identified by the isosurfaces of the second invariant $Q^{(\sigma)}$ of the velocity gradient tensor coarse-grained with $\sigma^{*}=0.2$. The threshold is $Q^{(\sigma) *}=16$. The grid width indicates the boundary layer thickness at the exit plane of each visualization.

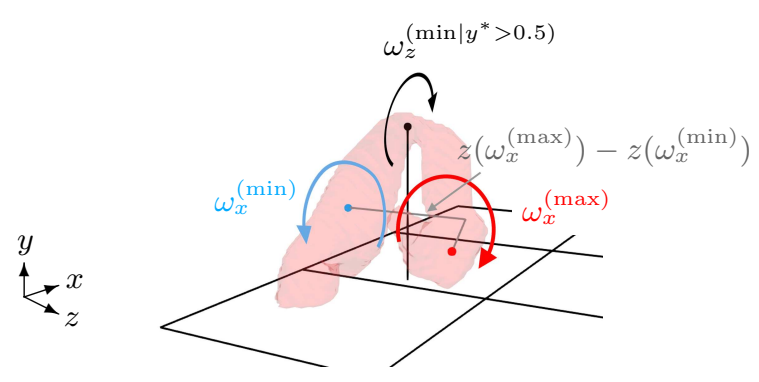

Fig. 10: An example of hairpin vortices identified by the conditions (2). The transparent isosurface with the threshold $Q^{(\sigma) *}=16$ is identical to the red one in Fig. $6(\mathrm{~b})$.

condition that the vortex has two streamwise legs and a head above $y^{*}=0.5$. The second condition $(2 \mathrm{~b})$ implies that the spanwise distance between the legs is larger than $2 \sigma\left(=0.4 \delta_{99}\right)$, which is indicated in Fig. 10 (an example of a hairpin vortex identified by these conditions) by the gray line between $\omega_{x}^{(\max )}$ (red point) and $\omega_{x}^{(\min )}$ (blue point). The third condition (2c) implies that the head $\omega_{z}^{\left(\min \mid y^{*}>0.5\right)}$ (black point) is located between the legs (red and blue points) in the spanwise direction. Figure 11 shows hairpin vortices (red) which satisfy these conditions (2) and $V^{*} \geq 0.01$ among vortices identified in Fig. 9. It is important that we can observe hairpin vortices irrespective of the Reynolds num- ber [(a) $990 \leq R e_{\theta} \leq 1950$ and (b) $\left.2900 \leq R e_{\theta} \leq 3780\right]$. The conditions (2) also identify vortices with short and long legs, that is, the so-called one-legged hairpin vortices.

In addition to the hairpin vortices, we also identify in Fig. 11 quasi-streamwise vortices (blue) which satisfy the following conditions:

$$
\begin{aligned}
& \omega_{x}^{(\max )} \omega_{x}^{(\min )}>0, \\
& \ell_{x}>\delta_{99},
\end{aligned}
$$

where $\ell_{x}$ is the streamwise length of the objects. The former condition (3a) implies that the sign of the streamwise vorticity in an individual vortex $\left(Q^{(\sigma) *} \geq\right.$ 16) does not change. Namely, no vortices can simultaneously satisfy the conditions for hairpin (2a) and for quasi-streamwise vortices (3a). The latter condition $(3 \mathrm{~b})$ is that vortices are longer in the streamwise direction than the boundary layer thickness. Although we do not impose a condition for the inclination or the length in the wall-normal direction, most of the identified quasi-streamwise vortices are aligned in the direction of the mean-flow stretching (approximately 45 degrees in the log layer). This is consistent with the fact that vortices with the size $(\sigma \approx y)$ comparable to the height in the log layer are stretched by the mean shear (Motoori and Goto, 2019a).

By using these conditions (2) and (3), we can distinguish three types of vortices: hairpin vortices, quasi- 


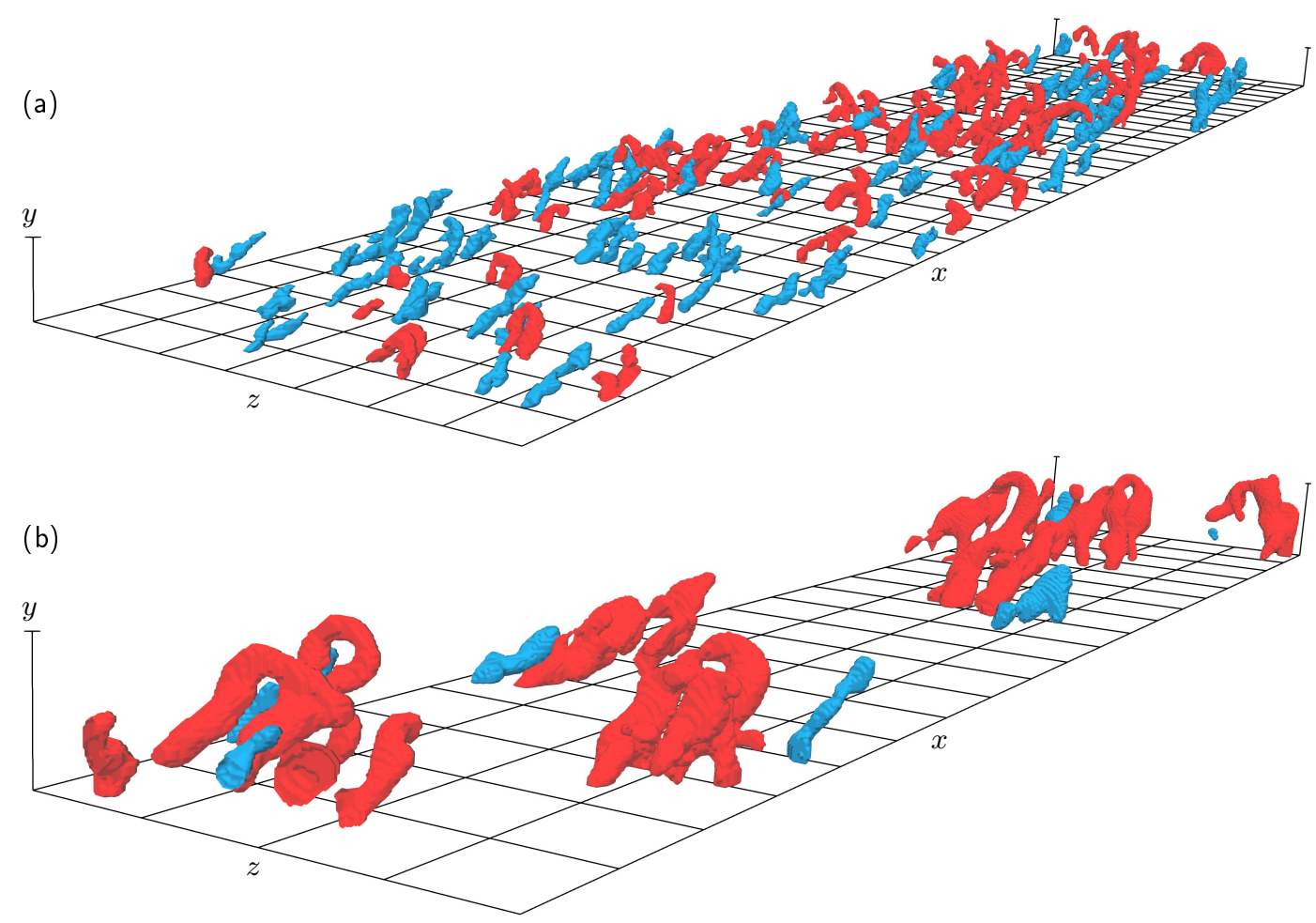

Fig. 11: Visualizations in the same regions (a) R3 and (b) R5 as in Fig. 9. We extract the largest-scale hairpin vortices (red) and quasi-streamwise vortices inclined to the wall (blue) under the conditions (2) and (3), respectively. The grid width indicates the boundary layer thickness at the exit plane of each visualization.

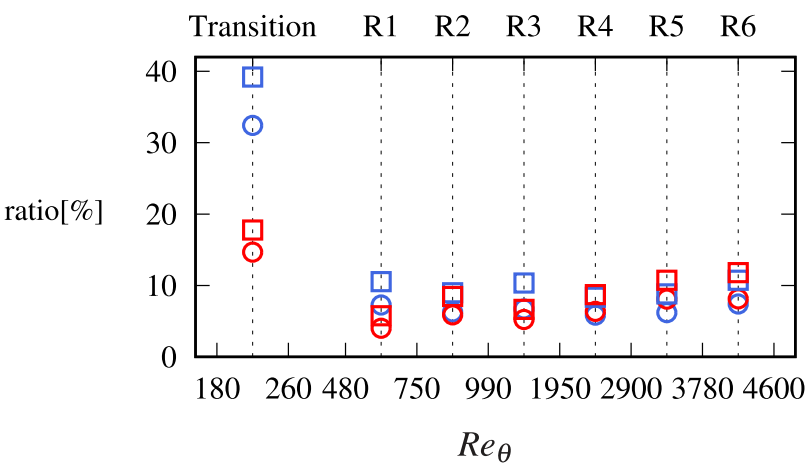

Fig. 12: The number ratios of hairpin (red) and quasistreamwise vortices (blue) to all the largest-scale vortices under the condition $V^{*} \geq 0.01$ (circles) or $V^{*} \geq 0.02$ (squares) in the developed turbulence (R1-R6; see Table 2) and the transition region $\left(R e_{\theta}=180-260\right)$ for the five snapshots.

streamwise vortices and the others. We show in Fig. 12 the number ratios of hairpin (red) and quasi-streamwise vortices (blue) to all the largest-scale vortices satisfying $V^{*} \geq 0.01$ (circles) and $V^{*} \geq 0.02$ (squares) in the six developed-turbulent-region R1-R6 (Table 2). We see that these ratios are almost constant irrespective of $R e_{\theta}$ and that the values under the condition $V^{*} \geq 0.02$ are larger than those under $V^{*} \geq 0.01$. This is a quantitative evidence that hairpin and quasi-streamwise vor- tices do not disappear in the downstream regions and they are in the largest scale of developed turbulence.

Note that, even in the transition region $\left(R e_{\theta}=180\right.$ 260) where artificial hairpin vortices are induced by the body force, the ratio of hairpin vortices is less than $20 \%$, whereas that of streamwise vortices has larger values $(30 \%-40 \%)$. This is consistent with the visualizations [Fig. 1 and Fig. 7a in Eitel-Amor et al. (2015)] of the transition region, in which streamwise vortices are frequently observed but they are developing into hairpin vortices. Although the values (approximately $15 \%-20 \%$ ) in the transition region are larger than those in the developed turbulence $(5 \%-10 \%)$, it is most important that they never decrease in R1-R6. In other words, hairpin vortices (and quasi-streamwise vortices) exist as developed three-dimensional structures in the largest scale of fully developed turbulent boundary layers.

\section{Discussion: hairpin vortex}

In the previous section, we have identified the largest-scale vortices by coarse-graining turbulent fields with the filter width comparable to the boundary layer thickness (Fig. 9). Then, we have quantitatively shown that hairpin vortices and quasi-streamwise vortices inclined to the wall exist in the largest-scale structures irrespective of the Reynolds number (Fig. 12). In this 
section, we show that these results are not conflicting with the visualizations of three-dimensional vortical structures in the previous DNS studies or with the observations of the HVS in the previous experiments.

In low-Reynolds-number regions where the transition effect remains, Wu and Moin (2009a,b) reported that hairpin vortices are dominant. There only exist single-scale vortices, that is, they are the largest but, at the same time, the smallest vortices. This is the reason why the largest-scale hairpin vortices are identified without coarse-graining. However, as the Reynolds number increases, the hierarchy of vortices appears. Recall that vortices identified by the velocity gradient without coarse-graining are at the smallest scale (see Figs. 3 and 4 in Motoori and Goto, 2019a). Therefore, the vortices in the $\log$ and outer layers identified by Jiménez et al. (2010) and Schlatter et al. (2014) are at the smallest scale and their shapes are not hairpin. This is not inconsistent with the result for the low Reynolds number (Wu and Moin, 2009a,b) or the present observation that hairpin vortices exist in the largest-scale field irrespective of the Reynolds number. Here, we reemphasize that we cannot discuss buffer-layer structures in developed turbulence because we filter them out.

In many DNS studies (Jiménez et al., 2010; Schlatter et al., 2014; Eitel-Amor et al., 2015; Rahgozar and Maciel, 2016), not hairpin vortices but arch-like vortices were observed in the outer layer of the turbulent fields without coarse-graining. This does not conflict with the present observation. Since, in the outer layer, there is no scale separation, the arch-like vortices can be identified in the turbulent fields without coarse-graining. However, in the log layer, where a hierarchy of multiscale vortices exists, the largest-scale vortices tend to be streamwisely elongated and their downstream parts are lifted up (Fig. 9). The largest-scale streamwise vortices cannot be identified without coarse-graining, and only the arch-like heads of the hairpin vortices are observed.

In contrast to the identification in terms of the velocity gradients, the HVS identifies hairpin vortices in terms of the velocity (ejections, sweeps and low-speed regions). Since the largest-scale structures (i.e. the hairpin vortices) are relevant to these motions, it is natural to observe the largest-scale hairpin vortices by the criteria (Adrian et al., 2000; Ganapathisubramani et al., 2003; Adrian, 2007). The multiscale nature thus explains that the present result is consistent with the previous numerical and experimental results.

Before closing this section, we show in Fig. 13 the largest-scale vortices and low-speed regions identified by the blue isosurfaces of the fluctuating streamwise velocity $u^{(\sigma) *}=-1$ coarse-grained at $\sigma^{*}=0.2$. We see that the largest-scale low-speed regions (i.e. LSM) are streamwisely elongated, and they are located between the legs of the hairpin vortices or beside quasistreamwise vortices. This observation is consistent with

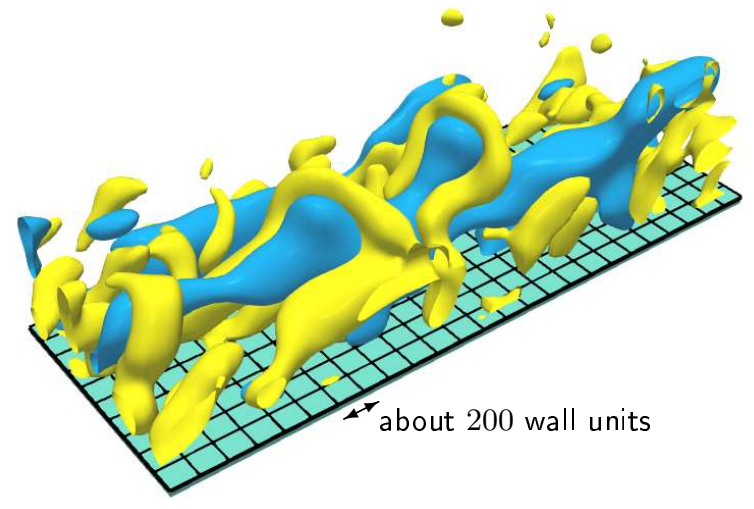

Fig. 13: Largest-scale vortices (yellow) and low-speed regions (blue) identified by the isosurfaces of $Q^{(\sigma) *}=16$ and $u^{(\sigma) *}=$ -1 , respectively. A subdomain $\left(\operatorname{Re}_{\theta} \approx 3000\right)$ in Fig. $9(\mathrm{~b})$ is shown.

the result by Kevin et al. (2019a) that the conditional averaged velocity fields are one-sided-rotating (quasistreamwise vortices) or counter-rotating flow (hairpin vortices). We also see that these vortices form packets along the LSM, which is also consistent with the observations of LSM by PIV (e.g. Adrian et al., 2000; Ganapathisubramani et al., 2003; Adrian, 2007; Dennis and Nickels, 2011a,b) and DNS (e.g. Lee and Sung, 2011; Lee et al., 2014). It is also interesting that this snapshot is reminiscent of the schematic of coherent vortices drawn by Adrian et al. (2000). The picture is therefore evidence supporting their idea, though we have not discussed the hairpin vortices in the buffer and $\log$ layers of the developed turbulence. This issue of the dominance of the hierarchy of attached hairpin vortices is a target of our future studies, which is related to the experimental study by Jodai and Elsinga (2016).

\section{Conclusions}

We have conducted the DNS of a turbulent boundary layer for $R e_{\theta}=180-4600$ and investigated vortices at the largest scale with the size comparable to the boundary layer thickness. The key to the present analyses is to identify the largest-scale vortices in an objective manner in the turbulent fields coarse-grained at the boundary-layer-thickness scale. The most important conclusion is that hairpin vortices never disappear in the downstream regions and they exist in the largestscale structures.

In the present study, we identify coherent vortices by the isosurfaces of the second invariant of the coarsegrained velocity gradient tensor but we objectively determine the threshold $\left(Q^{(\sigma) *}=16\right)$ on the basis of results of the percolation analyses (Fig. 7). The visualizations (Fig. 9) demonstrate that the largest-scale vortices are similar to coherent vortices in the lowReynolds-number regions, that is, hairpin and quasistreamwise vortices. The quasi-streamwise legs of the 
hairpin and the quasi-streamwise vortices tend to align to the direction of the mean-flow stretching. We then identify hairpin and quasi-streamwise vortices by the criteria (2) and (3) in terms of the coarse-grained vorticity. Qualitative evidence (Fig. 12) supports the visualization (Fig. 9) that hairpin vortices and quasistreamwise vortices exist in the largest-scale structures irrespective of the Reynolds number. These results are not conflicting with the previous results of the DNS and experiments (see Section 4). In addition, it is striking to observe that the visualization (Fig. 13) of the largestscale vortices and low-speed regions resembles the wellknown schematic drawn by Adrian et al. (2000).

\section{Acknowledgements}

The DNS was mainly conducted under the $\mathrm{K}$ computer provided by the RIKEN Advanced Institute for Computational Science through the HPCI System Research projects (hp170361 and 180257). The part of the DNS and their analyses were conducted under the auspices of the NIFS Collaboration Research Programs (NIFS18KNSS108). This work was also supported by JSPS Grants-in-Aid for Scientific Research 16H04268, $20 \mathrm{H} 02068$ and 20J10399.

\section{References}

Abe, H., Kawamura, H., Choi, H., 2004. Very large-scale structures and their effects on the wall shear-stress fluctuations in a turbulent channel flow up to $R e_{\tau}=640$. J. Fluids Engng $126,835$.

Adrian, R.J., 2007. Hairpin vortex organization in wall turbulence. Phys. Fluids 19, 041301.

Adrian, R.J., Meinhart, C.D., Tomkins, C.D., 2000. Vortex organization in the outer region of the turbulent boundary layer. J. Fluid Mech. 422, 1-54.

del Álamo, J.C., Jiménez, J., Zandonade, P., Moser, R.D., 2006. Self-similar vortex clusters in the turbulent logarithmic region. J. Fluid Mech. 561, 329-358.

Bandyopadhyay, P., 1980. Large structure with a characteristic upstream interface in turbulent boundary layers. Phys. Fluids $23,2326$.

Chong, M.S., Perry, A.E., Cantwell, B.J., 1990. A general classification of three-dimensional flow fields. Phys. Fluids A 2, $765-777$.

Dennis, D.J.C., Nickels, T.B., 2011a. Experimental measurement of large-scale three-dimensional structures in a turbulent boundary layer. Part 1. Vortex packets. J. Fluid Mech. 673, 180-217.

Dennis, D.J.C., Nickels, T.B., 2011b. Experimental measurement of large-scale three-dimensional structures in a turbulent boundary layer. Part 2. Long structures. J. Fluid Mech. 673, 218-244.

Deshpande, R., Monty, J.P., Marusic, I., 2019. Streamwise inclination angle of large wall-attached structures in turbulent boundary layers. J. Fluid Mech. 877, R4.

Eitel-Amor, G., Örlü, R., Schlatter, P., Flores, O., 2015. Hairpin vortices in turbulent boundary layers. Phys. Fluids 27, 025108.

Elsinga, G.E., Adrian, R.J., Van Oudheusden, B.W., Scarano, F., 2010. Three-dimensional vortex organization in a highReynolds-number supersonic turbulent boundary layer. J. Fluid Mech. 644, 35-60.
Falco, R.E., 1977. Coherent motions in the outer region of turbulent boundary layers. Phys. Fluids 20, S124.

Ganapathisubramani, B., Longmire, E.K., Marusic, I., 2003. Characteristics of vortex packets in turbulent boundary layers. J. Fluid Mech. 478, 35-46.

Head, M.R., Bandyopadhyay, P., 1981. New aspects of turbulent boundary-layer structure. J. Fluid Mech. 107, 297-338.

Hoshen, J., Kopelman, R., 1976. Percolation and cluster distribution. I. Cluster multiple labeling technique and critical concentration algorithm. Phys. Rev. B 14, 3438-3445.

Hutchins, N., Marusic, I., 2007. Evidence of very long meandering features in the logarithmic region of turbulent boundary layers. J. Fluid Mech. 579, 1-28.

Hwang, J., Lee, J., Sung, H.J., Zaki, T.A., 2016. Inner-outer interactions of large-scale structures in turbulent channel flow. J. Fluid Mech. 790, 128-157.

Hwang, Y., 2015. Statistical structure of self-sustaining attached eddies in turbulent channel flow. J. Fluid Mech. 767, 254-289.

Jeong, J., Hussain, F., 1995. On the identification of a vortex. J. Fluid Mech. 285, 69-94.

Jeong, J., Hussain, F., Schoppa, W., Kim, J., 1997. Coherent structures near the wall in a turbulent channel flow. J. Fluid Mech. 332, 185-214.

Jiménez, J., Hoyas, S., Simens, M.P., Mizuno, Y., 2010. Turbulent boundary layers and channels at moderate Reynolds numbers. J. Fluid Mech. 657, 335-360.

Jiménez, J., Moin, P., 1991. The minimal flow unit in near-wall turbulence. J. Fluid Mech. 225, 213-240.

Jodai, Y., Elsinga, G.E., 2016. Experimental observation of hairpin auto-generation events in a turbulent boundary layer. J. Fluid Mech. 795, 611-633.

Kevin, Monty, J., Hutchins, N., 2019a. The meandering behaviour of large-scale structures in turbulent boundary layers. J. Fluid Mech. 865, R1.

Kevin, Monty, J., Hutchins, N., 2019b. Turbulent structures in a statistically three-dimensional boundary layer. J. Fluid Mech. 859, 543-565.

Kline, S.J., Reynolds, W.C., Schraub, F.A., Runstadler, P.W., 1967. The structure of turbulent boundary layers. J. Fluid Mech. 30, 741-773.

Lee, J., Ahn, J., Sung, H.J., 2015. Comparison of large- and very-large-scale motions in turbulent pipe and channel flows. Phys. Fluids 27, 025101.

Lee, J., Jung, S.Y., Sung, H.J., Zaki, T.A., 2013. Effect of wall heating on turbulent boundary layers with temperaturedependent viscosity. J. Fluid Mech. 726, 196-225.

Lee, J., Lee, J.H., Choi, J.I., Sung, H.J., 2014. Spatial organization of large- and very-large-scale motions in a turbulent channel flow. J. Fluid Mech. 749, 818-840.

Lee, J., Sung, H.J., Zaki, T.A., 2017. Signature of large-scale motions on turbulent/non-turbulent interface in boundary layers. J. Fluid Mech. 819, 165-187.

Lee, J.H., Sung, H.J., 2011. Very-large-scale motions in a turbulent boundary layer. J. Fluid Mech. 673, 80-120.

Lozano-Durán, A., Flores, O., Jiménez, J., 2012. The threedimensional structure of momentum transfer in turbulent channels. J. Fluid Mech. 694, 100-130.

Lozano-Durán, A., Holzner, M., Jiménez, J., 2016. Multiscale analysis of the topological invariants in the logarithmic region of turbulent channels at a friction Reynolds number of 932. J. Fluid Mech. 803, 356-394.

Marusic, I., Monty, J.P., 2019. Attached eddy model of wall turbulence. Annu. Rev. Fluid Mech. 51, 49-74.

Motoori, Y., Goto, S., 2019a. Generation mechanism of a hierarchy of vortices in a turbulent boundary layer. J. Fluid Mech. $865,1085-1109$.

Motoori, Y., Goto, S., 2019b. Scale-dependent enstrophy production rates in a turbulent boundary layer. J. Fluid Sci. Technol. 
14, JFST0016.

Osawa, K., Jiménez, J., 2018. Intense structures of different momentum fluxes in turbulent channels. Phys. Rev. Fluids 3, 084603.

Perry, A.E., Chong, M.S., 1982. On the mechanism of wall turbulence. J. Fluid Mech. 119, 173-217.

Perry, A.E., Henbest, S., Chong, M.S., 1986. A theoretical and experimental study of wall turbulence. J. Fluid Mech. 165, 163-199.

Perry, A.E., Marusic, I., 1995. A wall-wake model for the turbulence structure of boundary layers. Part 1. Extension of the attached eddy hypothesis. J. Fluid Mech. 298, 361-388.

Rahgozar, S., Maciel, Y., 2016. A visual assessment of hairpin packet structures in a DNS of a turbulent boundary layer. Eur. J. Mech. B/Fluids 56, 161-171.

Robinson, S., 1991. Coherent motions in the turbulent boundary layer. Annu. Rev. Fluid Mech. 23, 601-639.

Schlatter, P., Li, Q., Örlü, R., Hussain, F., Henningson, D.S., 2014. On the near-wall vortical structures at moderate Reynolds numbers. Eur. J. Mech. B/Fluids 48, 75-93.

Schlatter, P., Örlü, R., 2010. Assessment of direct numerical simulation data of turbulent boundary layers. J. Fluid Mech. 659, 116-126.

Smith, C.R., Walker, J.D.A., Haidari, A.H., Sobrun, U., 1991. On the dynamics of near-wall turbulence. Philos. Trans. R. Soc. London, Ser. A 336, 131.

Spalart, P.R., 1988. Direct simulation of a turbulent boundary layer up to $R_{\theta}=1410$. J. Fluid Mech. 187, 61-98.

Theodorsen, T., 1952. Mechanism of turbulence, in: Proceedings of the Midwest Conference on Fluid Mechanics, Ohio State University.

Theodorsen, T., 1955. The structure of turbulence, in: 50 Jahre Grenzschictforschung, edited by H. Gortler and W. Tollmein, Friedrich Vieweg \& Sohn, Braunschweig.

Townsend, A.A., 1976. The Structure of Turbulent Shear Flow. Cambridge University Press.

Wu, X., Moin, P., 2009a. Direct numerical simulation of turbulence in a nominally zero-pressure-gradient flat-plate boundary layer. J. Fluid Mech. 630, 5-41.

Wu, X., Moin, P., 2009b. Forest of hairpins in a lowReynolds-number zero-pressure-gradient flat-plate boundary layer. Phys. Fluids 21, 091106. 